Pathophysiology of Haemostasis and Thrombosis

\title{
Impaired Flow-Mediated Vasodilation in vivo and Reduced Shear-Induced Platelet Reactivity in vitro in Response to Nitric Oxide in Prothrombotic, Stroke-Prone Spontaneously Hypertensive Rats
}

\author{
Tomomi Taka $^{a}$ Yoshio Ohta $^{\mathrm{b}}$ Junji Sekic John C. Giddings ${ }^{d}$ \\ Junichiro Yamamoto ${ }^{a}$ \\ a Laboratory of Physiology, Faculty of Nutrition and High Technology Research Centre, Kobe Gakuin University, \\ Kobe, b Nara Hospital, Kinki University School of Medicine, Nara, cDepartment of Biomedical Engineering, \\ National Cardiovascular Centre Research Institute, Osaka, Japan, dDepartment of Haematology, \\ University of Wales, College of Medicine, Cardiff, UK
}

\section{Key Words}

SHRSP · Flow-mediated vasodilation · Nitric oxide •

Platelet · Shear · Hypertension

\begin{abstract}
Previous investigations using an He-Ne laser-induced thrombosis method have shown that stroke-prone spontaneously hypertensive rats (SHRSP) have an enhanced thrombotic tendency in vivo compared to normotensive, Wistar Kyoto rats (WKY). In addition, studies in the presence of acetylcholine have suggested the presence of endothelial dysfunction in SHRSP. In contrast, shearinduced platelet reactivity in vitro appeared to be depressed in SHRSP. The aim of the present study was to investigate endothelial function in SHRSP using a new physiological in vivo model, and to determine the response of platelets to nitric oxide (NO) in non-anticoagulated blood using a shear-induced platelet function in vitro method (haemostatometry). Endothelial function was estimated by measuring flow-mediated vasodilation (FMV) of the femoral artery. Vessels were exposed and
\end{abstract}

blood flow was arrested using a silicone-coated arterial clamp. Vasodilation was measured by computer-assisted image analysis $3 \mathrm{~min}$ after release of stasis. Arterial vasodilation was observed in the femoral artery of WKY, but not in SHRSP. Vasodilation was seen in both WKY and SHRSP; however, in response to the NO donor, 1-hydroxy-2-oxo-3-(3-aminopropyl)-3-isopropyl-1triazene (NOC 5). In contrast, $100 \mu$ M NOC 5 did not affect platelet reactivity in SHRSP. The NO scavenger, 2-(4-carboxyphenyl)-4,4,5,5-tetramethylimidazoline-1-oxyl 3-oxide, sodium salt (carboxy-PTIO) and the NO synthase inhibitor, $N^{G}$-nitro-L-arginine methyl ester, hydrochloride ( $L-N A M E$ ), did not affect shear-induced platelet reactivity. NOC 5 at $10 \mu M$ (final concentration) inhibited shear-induced platelet reactivity in WKY. These results confirm the presence of endothelial dysfunction in SHRSP and indicate that platelets are non-responsive to $\mathrm{NO}$ in this hypertensive model. The data suggest that defective endothelial reactions or disturbed thrombogenic mechanisms outweigh the platelet hyporeactivity and contribute to the prothrombotic status in SHRSP.

Copyright $\odot 2002$ S. Karger AG, Basel

\begin{tabular}{ll}
\hline KARGER & @ 2002 S. Karger AG, Basel \\
1424-8832/02/0324-0184\$18.50/0 \\
$\begin{array}{l}\text { Eax +4161306 1234 } \\
\text { www.karger.com }\end{array}$ & $\begin{array}{l}\text { Accessible online at: } \\
\text { www.karger.com/pht }\end{array}$
\end{tabular}

Tomomi Taka

Laboratory of Physiology, Faculty of Nutrition

Kobe Gakuin University

518 Arise Ikawadani-cho, Nishi-ku, Kobe 651-2180 (Japan)

Tel. +81 78974 1551, Fax +81 78974 5689, E-Mail tomomi_oishi@mbk.nifty.com 


\section{Introduction}

Arterial platelet-rich thrombogenesis plays an important role in life-style-related athero-thrombotic diseases such as stroke and myocardial infarction. The precise relationships between prothrombotic mechanisms and the development of cardiovascular disorders remain to be fully evaluated, however, and a number of useful animal models have been described for this purpose. In this respect, we have utilised a sensitive and reproducible He$\mathrm{Ne}$ laser-induced thrombosis technique to assess thrombotic status in vivo [1-3] and a well-defined test of primary haemostasis (haemostatometry) to analyse shear-induced platelet function in native, non-anticoagulated blood [4-8].

Using these techniques, we determined that one breed of congenitally diabetic rat (Goto-Kakizaki) appeared to have an enhanced thrombotic tendency in vivo and platelet hyper-aggregability in vitro compared to the control Wistar rat [9]. In contrast, another breed of rat with congenital diabetes (Otsuka Long-Evans Tokushima Fatty rat) appeared to have a reduced thrombotic tendency and platelet hypo-aggregability [10]. In these experiments, therefore, in vivo and in vitro reactions were consistent and suggested that platelet function contributed directly to thrombogenetic mechanisms. In stroke-prone spontaneously hypertensive rats (SHRSP), however, an enhanced thrombotic tendency in vivo was observed in association with depressed shear-induced platelet function $[11,12]$. These results demonstrated that the relationship between the in vivo and in vitro measurements was not straightforward in this animal model. In addition, assessment of He-Ne laser-induced thrombosis in SHRSP in the presence of acetylcholine suggested that endothelial dysfuntion prevailed over the platelet hypo-aggregability and mediated the enhanced thrombotic tendency in these animals [12]. Overall, therefore, the findings suggested that measurements of both platelet reactivity and endothelial function might be required to optimally investigate thrombotic status.

Endothelial dysfunction and NO metabolism are key processes in atherosclerosis and thrombosis [13]. Flowmediated vasodilation (FMV) is thought to reflect endothelial NO release, and techniques have been described using FMV to quantify endothelial function in humans [14-16]. In animals, however, tests of vasodilation have classically utilised ex vivo arterial segments $[17,18]$ and vasoactive responses have been studied in situ in only a small number of experiments $[19,20]$. The aim of the present study was to establish a physiologically relevant technique based on FMV to evaluate endothelial function in SHRSP in vivo, and to relate the findings to the effects of NO on shear-induced platelet function in this model.

\section{Materials and Methods}

\section{Reagents}

The NO scavenger, 2-(4-carboxyphenyl)-4,4,5,5-tetramethylimidazoline-1-oxyl 3-oxide, sodium salt (carboxy-PTIO), and the NO synthase (NOS) inhibitor, $N^{\mathrm{G}}$-nitro- $L$-arginine methyl ester, hydrochloride ( $L$-NAME), were dissolved and diluted in saline. The NO donor, 1-hydroxy-2-oxo-3-(3-aminopropyl)-3-isopropyl-1-triazene (NOC 5), was dissolved and diluted in $0.1 \mathrm{M} \mathrm{NaOH}$. The reagents were purchased from Dojindo Laboratories (Kumamoto, Japan). Solutions were stored at $-80^{\circ} \mathrm{C}$ and used within 2 weeks of preparation.

\section{Animals}

Male SHRSP and normotensive WKY rats were kindly donated by Kinki University School of Medicine (Osaka, Japan) at 8-20 weeks of age. Animals were fed a commercial solid diet (SP diet, Funabashi farm, Japan) and given tap water ad libitum. They were housed in compliance with the 'Guiding Principles for the Care and Use of Animals in the Field of Physiological Sciences' published by the Physiological Society of Japan. All experiments were performed at 28-31 weeks of age (body weight in WKY and SHRSP rats, 451.5 \pm 6.1 vs. $322.5 \pm 8.8 \mathrm{~g}, \mathrm{p}<0.001$; systolic blood pressure, $166.8 \pm$ 7.0 vs. $240.4 \pm 7.4 \mathrm{~mm} \mathrm{Hg}, \mathrm{p}<0.001$, respectively).

\section{Flow-Mediated Vasodilation}

Animals were anesthetized with sodium pentobarbital $(60 \mathrm{mg} / \mathrm{kg}$, i.m.). They were artificially ventilated and placed on a heated pad to maintain body temperature. The left femoral artery was carefully exposed and removed from the surrounding tissue. The vessel was compressed at a proximal part of the exposed artery using an arterial clamp coated with silicon tubing. Complete occlusion was established gently and maintained for $3 \mathrm{~min}$. The clamp was removed to restore blood flow and changes in vessel diameter were monitored 2-4 mm distal from the site of occlusion.

The animals were left undisturbed for $30 \mathrm{~min}$ before the exposed arteries were bathed in NOC 5 solution to investigate endotheliumindependent dilation stimulated by exogenous NO. The final concentration of NOC 5 was approximately $5 \mu M$. Vessel diameters were monitored before and after treatment with the NO donor.

Changes in vessel diameter were continuously recorded on a videotape recorder. Subsequently, images at 30 -second intervals were transferred to a personal computer and vessel outer diameters were analysed with Image-Pro Plus software (version 4.0, Media Cybernetics, Inc., Md., USA). Baseline values were obtained $30 \mathrm{~s}$ before vessel occlusion or adding NOC 5. Changes in vessel diameter were expressed as a percentage of the baseline.

\section{In vitro Shear-Induced Platelet Reactivity Test}

(Haemostatometry)

A three-channel purpose-built haemostatometer was constructed in the Physiology Laboratory of the Faculty of Nutrition at Kobe Gakuin University. Details of the measurement of shear-induced platelet reactivity and dynamic coagulation (haemostatometry) have 
been described elsewhere [6-8]. In brief, blood was withdrawn using a 20-gauge needle, without anticoagulant, from the abdominal aorta of SHRSP or WKY rats anaesthetised with nembutal $(60 \mathrm{mg} / \mathrm{kg}$, i.m.). Blood was collected in $1.5-\mathrm{ml}$ aliquots into syringes containing 15 or $150 \mu \mathrm{l}$ of reagent or vehicle. After gentle inversion three times, measurements commenced without delay. Perfusion of the blood samples through standardised polyethylene tubing (outer diameter: $1.00 \pm 0.02 \mathrm{~mm}$; inner diameter: $0.50 \pm 0.01 \mathrm{~mm}$ ) was achieved by pumping paraffin liquid into the blood sample at a constant rate of $0.057 \mathrm{ml} / \mathrm{min}$. After $150 \mathrm{~s}$, pressure was stabilised at $60 \mathrm{~mm} \mathrm{Hg}$ and holes were punched in the tube using a needle (diameter: $0.18 \mathrm{~mm}$ ). Blood loss into surrounding saline led to the formation of plateletrich haemostatic plugs, blood coagulation and subsequent arrest of blood flow. Pressure changes were monitored and the parameters $\mathrm{H} 1$ and $\mathrm{H} 2(\mathrm{~mm} \mathrm{Hg} \cdot \mathrm{s}), \mathrm{CT} 1$ and CT2 (min) were defined as indices of platelet reactivity (adhesion/aggregation) and dynamic coagulation, respectively.

Measurement of Blood Pressure

Systolic blood pressures were measured by the tail cuff method (UR-1000, Ueda Seisakusyo, Tokyo, Japan).

\section{Statistical Analysis}

All data are presented as mean \pm SEM. The $\mathrm{H} 1$ and $\mathrm{H} 2$ parameters obtained by haemostatometry were converted to logarithms before statistical analysis. Results were analysed by the unpaired $t$ test, one-way factorial ANOVA or one-way repeated-measures ANOVA, followed by post-hoc test (Fisher's PLSD) using the commercially available statistical package Stat View (version 5.0; SAS Institute Inc., Cary, N.C., USA). $\mathrm{p}<0.05$ was considered to be statistically significant.

\section{Results}

Vasodilation Induced by Flow and Exogenous NO Comparison of SHRSP and WKY

Vessel diameters in WKY after occlusion for $3 \mathrm{~min}$ were significantly increased compared to baseline. In contrast, vessel diameters in SHRSP were not changed after temporary vessel occlusion. Thus, FMV was evident in the control rats but not in the hypertensive animals (fig. 1a, p < 0.0001). Exogenous NOC 5-induced vessel dilation in both SHRSP and WKY rats $(109.8 \pm 1.7 \%$ and $109.6 \pm 3.0 \%$, at $30 \mathrm{~s}$ after NOC 5 adding). The changes mediated by NOC 5 were not significantly different between SHRSP and WKY (fig. 1b).

\section{Effects of NOC 5, Carboxy-PTIO and L-NAME on \\ Shear-Induced Platelet Reactivity and Dynamic \\ Coagulation}

The effects of the NO scavenger, carboxy-PTIO, and the NOS inhibitor, $L$-NAME on shear-induced platelet reactivity and dynamic coagulation were assessed by haemostatometry in WKY rat (fig. 2). H1 and H2, and, CT1

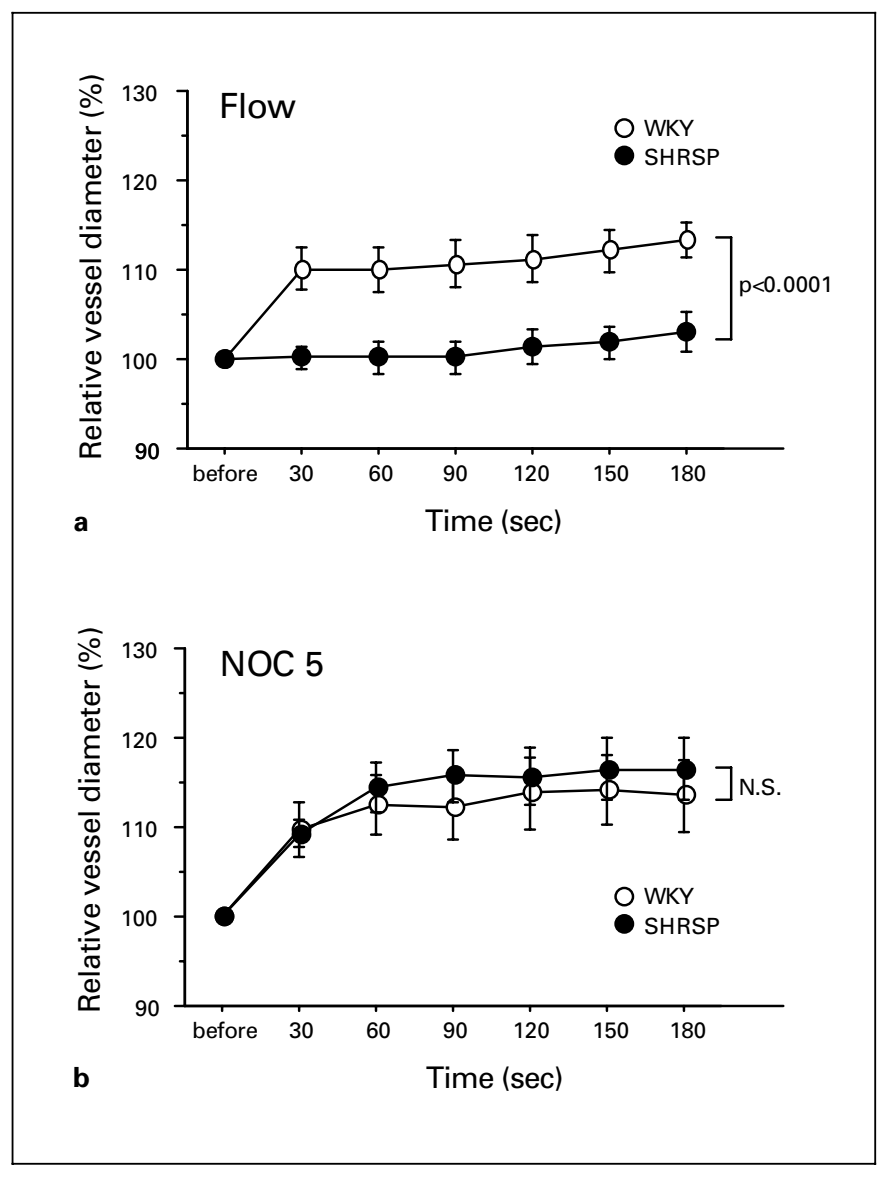

Fig. 1. Changes in femoral arterial diameters in response to an increase in blood flow after temporary vessel occlusion (a) and treatment with NOC 5 (b) in WKY and SHRSP. Vessel diameters were measured $30 \mathrm{~s}$ before and every 30 -second interval after occlusion for 3 min or treatment with NOC 5 (final concentration of approximately $5 \mu M$ ). Vasodilation was significantly increased after restoration of flow in WKY but not in SHRSP. The vasodilation response was observed in both WKY and SHRSP after treatment with NOC 5. Values are means \pm SEM. $n=6-8$ rats in each group. NS $=$ Not significant.

and CT2, respectively, showed good correlation. Representative data for $\mathrm{H} 2$ and CT2 are illustrated in figure 2. Carboxy-PTIO did not affect shear-induced platelet reactivity but inhibited dynamic coagulation at concentration of $1 \mathrm{~m} M . L$-NAME had no effect on either shear-induced platelet reactivity or dynamic coagulation.

The NO donor, NOC 5, significantly and dose-dependently inhibited platelet reactivity at a concentration of $10 \mu M(\mathrm{p}<0.01)$ in WKY rats. It had no effect on dynamic coagulation in WKY. Neither platelet reactivity nor dynamic coagulation was affected by NOC-5 in 
Fig. 2. The effects of carboxy-PTIO and $L$-NAME on shear-induced platelet reactivity (H2) and dynamic coagulation (CT2) in WKY rats measured by haemostatometry. Neither agent affected shear-induced platelet reactivity. Carboxy-PTIO at a concentration of $1 \mathrm{~m} M$ inhibited dynamic coagulation. Values are means \pm SEM. $n=4-6$ rats in each group. ${ }^{* *} \mathrm{p}<0.01$ vs. control.

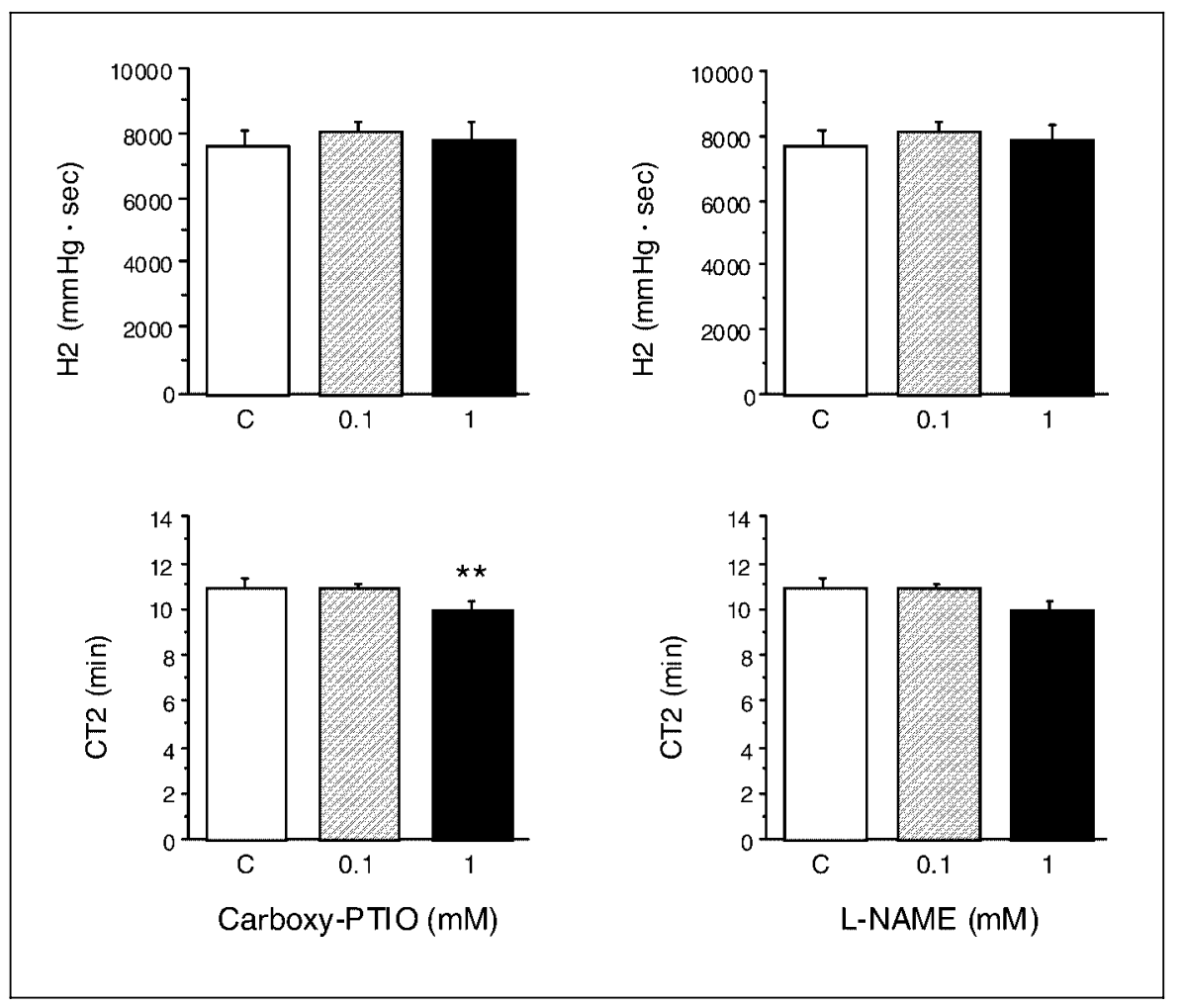

SHRSP. Basal shear-induced platelet reactivity in SHRSP was significantly lower than that in WKY $(p<0.001)$ (fig. 3).

\section{Discussion}

The classical Virchow's triad hypothesis describes interactions between blood cells, endothelial cells and blood flow as central to the pathogenesis of thrombosis. Moreover, recent investigations have clarified the role of blood flow or shear forces in endothelial-related mechanisms and have emphasized the relevance of physiological calcium and thrombin to thrombogenesis [21-24]. It is clear,

Fig. 3. The effects of NOC 5 on shear-induced platelet reactivity (H2) and dynamic coagulation (CT2) measured by haemostatometry. NOC 5 at a concentration of $10 \mu M$ significantly inhibited platelet reactivity in WKY but not in SHRSP. Dynamic coagulation was not changed by NOC 5 in either strain. The basal value of $\mathrm{H} 2$ was significantly lower in SHRSP than in WKY. Values are means \pm SEM. $\mathrm{n}=$ 9-11 rats in each group. ${ }^{*} \mathrm{p}<0.05 ;{ }^{* *} \mathrm{p}<0.01 ;{ }^{* * *} \mathrm{p}<0.001$.

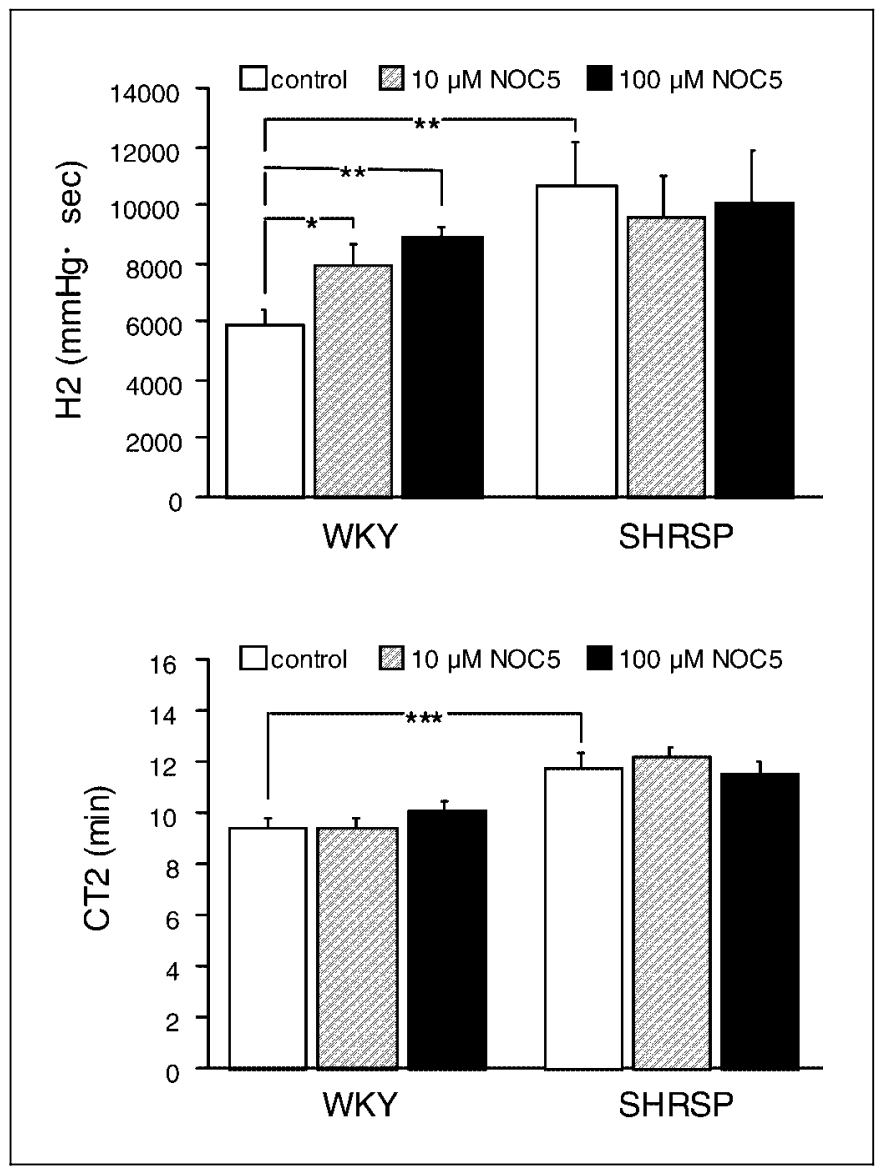

Pathophysiol Haemost Thromb 2002;32:184-189 
therefore, that the principles of the tests employed to fully evaluate thrombotic mechanisms in vivo should take account of these structural and functional relationships. In this respect, studies of experimental arterial plateletrich thrombi in animals provide a good approximation of pathophysiological processes and can be utilised to assess the effects of antithrombotic agents in vivo. Studies of this nature are not applicable to humans, however. Platelets play a central role in arterial thrombogenesis, and platelet function has been examined by various in vitro tests. The technique of haemostatometry has been developed to assess platelets activated by high shear forces in non-anticoagulated blood and has been used to determine thrombotic tendency in man under various conditions [4-6, 8, 25]. Gorog and Kovacs [26] proposed that the H1 parameter is an index of ADP-induced and that $\mathrm{H} 2$ reflects thrombin-induced platelet mechanisms. Later experiments have shown, however, that this distinction might not be totally valid. Therefore, both $\mathrm{H} 1$ and $\mathrm{H} 2$ are used as indices of general platelet reactivity. The CT1 and CT2 parameters identify stages of the blood coagulation process [7]. Antiplatelet agents inhibit only platelet reactivity (H1 and H2) but not coagulation (CT1 and CT2) [7]. In addition, shear-induced haemostasis tests such as haemostatometry are physiologically relevant to platelet reactivity in vivo $[6,27]$ and haemostatometry is very sensitive to physiological agonists compared to conventional tests. For example, unlike conventional platelet aggregometry, haemostatometry responds to physiological, nanomolar concentrations of norepinephrine [8]. As with the majority of in vitro platelet function tests, however, this method lacks the ability to examine the role of endothelial dysfunction in thrombogenesis.

An FMV test has been described to evaluate endothelial function in humans [14-16] in which vascular responses are mediated by endothelial derived NO [19]. In animals, endothelial function is classically assessed using excised aortic rings to which vasoactive agents may be added in culture chambers $[17,18]$. There are few reports using blood vessels in situ for evaluating endothelial function in animal models $[19,20]$. In the present study we have adapted the principles of FMV to examine endothelial function in SHRSP, a well-characterized prothrombotic model. Using exposed femoral arteries, we demonstrated that vasodilation occurred after the release of temporary stasis, and that this vasodilation lasted more than $15 \mathrm{~min}$. This appeared to be different from the results of human FMV tests in which the responses are transient and last for $180 \mathrm{~s}$ at most. These differences remain to be fully investigated but may be related to the surgical proce- dure performed in animals. Nevertheless, vasodilation consistently occurred in control animals (WKY) but not in SHRSP. In contrast, the NO donor, NOC 5, promoted vasodilation in SHRSP as well as in WKY, and it seems likely that this was an effect of NOC 5 on the smooth muscle layer rather than on the endothelium. These results were in keeping with our previous report that acetylcholine was not antithrombotic in SHRSP [12] and confirm the presence of endothelial dysfunction in the hypertensive animal. Similar results were also obtained in another hypertensive model (SHR) [28, 29], and the findings are supported by the observation that production of $\mathrm{NO}$ is decreased in hypertensive rats and human patients [11, 30, 31].

NO inhibits platelet reactivity (adhesion and aggregation) $[32,33]$ and there is evidence that impaired endothelial NO synthesis contributes to the development of arterial disease [34-36]. In the present study, the effects of an exogenous NO donor (NOC 5), an NO scavenger (carboxy-PTIO) and an NO synthase inhibitor ( $L$-NAME) were assessed using haemostatometry. Carboxy-PTIO and $L$-NAME did not affect these global haemostasis measurements. It may be that although the tests were completed within 4 min of blood collection, the short halflife of NO prevented detection of the effects of the NO modulators. In contrast, however, NOC 5 inhibited platelet reactivity in WKY, but not in SHRSP. These findings may reflect platelet-vessel wall reactions in vivo, and suggest that platelets in SHRSP are non-responsive to NO in the absence of endogenous endothelial NO release. Reduced platelet reactivity was observed in SHRSP. This might represent a protective phenomenon against the high thrombogenicity of the SHRSP vessel wall. There are reports showing the presence of NO synthase in platelets [37]. The present results, however, question the importance of platelet-derived NO in thrombogenesis.

In conclusion, we have established a new technique for evaluating endothelial function in situ in an animal model by adapting the principles of the well-characterized, FMV test previously used in humans. The present findings extend earlier data on the thrombotic tendency in the presence of endothelial disturbances observed in SHRSP. The method is especially useful to investigate the vascular endothelial contribution to thrombotic mechanisms in vivo.

\section{Acknowledgment}

We are grateful to Drs. T. Yamashita and Y. Sasaki, Mr. H. Ikarugi and Ms. R. Aoki for their valuable discussions. 


\section{References}

1 Kovacs IB, Tigyi-Sebes A, Trombitas K, Gorog P: Evans blue: An ideal energy-absorbing material to produce intravascular microinjury by He-Ne gas laser. Microvasc Res 1975;10:107124.

2 Yamamoto J, Iizumi H, Hirota R, Shimonaka K, Nagamatsu Y, Horie N, Morita S: Effect of physical training on thrombotic tendency in rats: Decrease in thrombotic tendency measured by the He-Ne laser-induced thrombus formation method. Haemostasis 1989;19:260265.

3 Hashimoto M, Yamashita T, Oiwa K, Watanabe S, Giddings JC, Yamamoto J: Enhancement of endogenous plasminogen activatorinduced thrombolysis by argatroban and APC and its control by TAFI, measured in an arterial thrombolysis model in vivo using rat mesenteric arterioles. Thromb Haemost 2002;87: 110-113.

4 Gorog P, Ahmed A: Haemostatometer: A new in vitro technique for assessing haemostatic activity of blood. Thromb Res 1984;34:341357.

5 Kovacs IB, Hutton RA, Kernoff PB: Hemostatic evaluation in bleeding disorders from native blood. Clinical experience with the hemostatometer. Am J Clin Pathol 1989;91:271-279.

6 Ratnatunga CP, Edmondson SF, Rees GM, Kovacs IB: High-dose aspirin inhibits shearinduced platelet reaction involving thrombin generation. Circulation 1992;85:1077-1082.

7 Yamamoto J, Taka T, Nakajima S, Ueda M, Sugimoto E, Sasaki Y, Muraki T, Seki J, Watanabe $\mathrm{S}$ : A shear-induced in vitro platelet function test can assess clinically relevant antithrombotic effects. Platelets 1999;10;178-184.

8 Ikarugi H, Taka T, Nakajima S, Noguchi T, Watanabe S, Sasaki Y, Haga S, Ueda T, Seki J, Yamamoto J: Norepinephrine, but not epinephrine, enhances platelet reactivity and coagulation after exercise in humans. J Appl Physiol 1999;86:133-138.

9 Taka T, Ono H, Sasaki Y, Seki J, Yamamoto J: Platelet reactivity in spontaneously diabetic rats is independent from blood glucose and insulin levels. Platelets 2002;13:313-316.

10 Taka T, Okita N, Sasaki Y, Yamamoto J: Thrombotic tendency of a non-insulin dependent diabetic rat OLETF. Research Conference on OLETF Rats 1996;2:75-79.

11 Noguchi T, Sasaki Y, Seki J, Giddings JC, Yamamoto J: Enhanced thrombogenicity and altered hemodynamics in the cerebral microvasculature of stroke-prone spontaneously hypertensive rats. Haemostasis 1997;27:237245 .
12 Yamashita T, Taka T, Nojima R, Ohta Y, Seki $\mathrm{J}$, Yamamoto $\mathrm{J}$ : There is no valid evidence presented as to an impaired endothelial NO system in the stroke-prone spontaneously hypertensive rats. Thromb Res 2002;105:507-511.

13 Ross R: The pathogenesis of atherosclerosis: A perspective for the 1990s. Nature 1993;362: 801-809.

14 Celermajer DS, Sorensen KE, Bull C, Robinson J, Deanfield JE: Endothelium-dependent dilation in the systemic arteries of asymptomatic subjects relates to coronary risk factors and their interaction. J Am Coll Cardiol 1994;24: 1468-1474.

15 Higashi Y, Sasaki S, Nakagawa K, Matsuura H, Kajiyama G, Oshima T: A noninvasive measurement of reactive hyperemia that can be used to assess resistance artery endothelial function in humans. Am J Cardiol 2001;87: 121-125.

16 Doshi SN, McDowell IFW, Moat SJ, Lang D, Newcombe RG, Kredan MB, Lewis MJ, Goodfellow J: Folate improves endothelial function in coronary artery disease. An effect mediated by reduction of intracellular superoxide? Arterioscler Thromb Vasc Biol 2001;21:1196-1202.

17 Onda T, Mashiko S, Hamano M, Tomita I, Tomita T: Enhancement of endothelium-dependent relaxation in the aorta from strokeprone spontaneously hypertensive rats at developmental stages of hypertension. Clin Exp Pharmacol Physiol 1994;21:857-863.

18 Tesfamariam B, Ogletree ML: Dissociation of endothelial cell dysfunction and blood pressure in SHR. Am J Physiol 1995;269:H189-H194.

19 Friebel M, Klotz KF, Ley K, Gaehtgens P, Pries AR: Flow-dependent regulation of arteriolar diameter in rat skeletal muscle in situ: Role of endothelium-derived relaxing factor and prostanoids. J Physiol 1995;483:715-726.

20 Pohl U, Holtz J, Busse R, Bassenge E: Crucial role of endothelium in the vasodilator response to increased flow in vivo. Hypertension 1986;8: 37-44.

21 Sakariassen KS, Ottenhof-Rovers M, Sixma JJ: Factor VIII-von Willebrand Factor requires calcium for facilitation of platelet adherence. Blood 1984;63:996-1003.

22 Chow TW, Hellums JD, Moake JL, Kroll MH: Shear stress-induced von Willebrand factor binding to platelet glycoprotein Ib initiates calcium influx associated with aggregation. Blood 1992;80:113-120.

23 Harker LA, Hanson SR, Runge MS. Thrombin hypothesis of thrombus generation and vascular lesion formation. Am J Cardiol 1995;75: 12B-17B.
24 Yamashita T, Yamamoto J, Sasaki Y, Matsuoka A: The antithrombotic effect of low molecular weight synthetic thrombin inhibitors, argatroban and PPACK, on He-Ne laser-induced thrombosis in rat mesenteric microvessels. Thromb Res 1993;69:93-100.

25 Mayou SC, Kovacs IB, Ridler CD, Kirby JD: Hemostatic abnormalities in Sneddon's syndrome. Angiology 1992;66:649-656.

26 Gorog P, Kovacs IB: Modelling coronary thrombosis from nonanticoagulated human blood in vitro. Hematol Pathol 1990;4:43-52.

27 Nakajima S, Noguchi T, Taka T, Ueda T, Kaizu K, Fukamizu M, Fujita S, Tabuchi M, Yamamoto J: A global platelet test of thrombosis and thrombolysis detects a prothrombotic state in some patients with non-insulin dependent diabetes and in some patients with stroke. Platelets 2000;11:459-466.

28 Koller A, Huang A: Impaired nitric oxidemediated flow-induced dilation in arterioles of spontaneously hypertensive rats. Circ Res 1994;74:416-421.

29 Koller A, Huang A: Development of nitric oxide and prostaglandin mediation of shear stress-induced arteriolar dilation with aging and hypertension. Hypertension 1999;34: 1073-1079.

30 Chou TC, Yen MH, Li CY, Ding YA: Alterations of nitric oxide synthase expression with aging and hypertension in rats. Hypertension 1998;31:643-648.

31 Node K, Kitakaze M, Yoshikawa H, Kosaka H, Hori M: Reduced plasma concentrations of nitrogen oxide in individuals with essential hypertension. Hypertension 1997;30:405-408.

32 Moncada S, Palmer RM, Higgs EA: Nitric oxide: Physiology, pathophysiology, and pharmacology. Pharmacol Rev 1991;43:109-142.

33 de Graaf JC, Banga JD, Moncada S, Palmer RM, de Groot PG, Sixma JJ: Nitric oxide functions as an inhibitor of platelet adhesion under flow conditions. Circulation 1992;85:22842290 .

34 Loscalzo J: Nitric oxide insufficiency, platelet activation, and arterial thrombosis. Circ Res 2001;88:756-762.

35 Sasaki Y, Seki J, Giddings JC, Yamamoto J: Effects of NO-donors on thrombus formation and microcirculation in cerebral vessels of the rat. Thromb Haemost 1996;76:111-117.

36 Boger RH, Bode-Boger SM, Thiele W, Junker W, Alexander K, Frölich JC: Biochemical evidence for impaired nitric oxide synthesis in patients with peripheral arterial occlusive disease. Circulation 1997;95:2068-2074.

37 Radomski MW, Palmer RM, Moncada S: An $L$-arginine/nitric oxide pathway present in human platelets regulates aggregation. Proc Natl Acad Sci USA 1990;87:5193-5197. 